\title{
Non-covalent Interactions Directed Rearrangement of Lactones to Pyrones: A Computational Study on the Mechanistic Insights of Solvent Assisted Gold(I) Catalyzed Reactions
}

\author{
G. Gayatri* \\ Department of Chemistry, Bharat Institute of Engineering and Technology, Ibrahimpatnam, \\ Telangana State, India
}

Received 25 July 2020, accepted in final revised final revised form 11 October 2020

\begin{abstract}
The mechanism of gold(I) catalyzed cycloisomerization of lactones to pyrones was analyzed by performing DFT study using B3LYP method with LanL2DZ basis set for Au and 6$31 \mathrm{~g}(\mathrm{~d})$ basis set for all other atoms. The potential energy surface of the reaction was scanned to know the preferred path for the formation of products under gas phase. The effects of solvent using acetonitrile, dichloromethane and toluene are explored as these solvents are reported to play a crucial role in the final product formation. The crossover between the oxophilic and alkynophilic reaction complexes, R-O and R-C, is found to be the major driving force which directs the product formation. While the larger energy gap between R-O and R-C leads to pyrone formation, the smaller difference and in turn the rapid crossover between R-O and R-C leads to the formation of decarboxylation adduct. Counterion plays a major role throughout the reaction. The atoms in molecules analysis on R-C using implicit as well as explicit solvent gave reason for the observed experimental trends and suggest that the prevailed non-covalent interactions play a substantial role in the product formation.
\end{abstract}

Keywords: Gold catalyzed reactions; Carbophilic, Oxophilic; Non-covalent interactions; Counterion; Cycloisomerization.

(C) 2021 JSR Publications. ISSN: 2070-0237 (Print); 2070-0245 (Online). All rights reserved. doi: http://dx.doi.org/10.3329/jsr.v13i1.48371 J. Sci. Res. 13 (1), 253-262 (2021)

\section{Introduction}

Gold ( $\mathrm{Au}$ ) catalyzed reactions have emerged as a key source for carrying out unprecedented cycloisomerization reactions for the past few decades due to the propensity of gold to bond with heteroatoms like -O, -N, -S [1] and has vast number of applications [2]. Au has a propensity to act as a soft and carbophilic Lewis acid and to activate $\mathrm{C}-\mathrm{C} \pi$ bonds. [1,3-5]. The mechanism involved in the formation of five and six membered rings via cycloisomerization paves a way to unravel the intricate details that underlie in the

*Corresponding author: gayathrig10@gmail.com 
course of reaction [6-10]. The presence of counter ion [11,12] and solvent considered [1315] plays a key role in driving the reaction process. Understanding the mechanism of such reactions is highly challenging and computational studies have always enlightened the chemists to unravel the mechanistic insights for such reactions [9,16-20]. Reports have shown the importance of non-covalent interactions in directing the reaction path and thereby exemplifying the product formation [21-23]. Metal-ligand complexes are also reported to involve in the antimicrobial activity suggesting new insights in this area [24]. Pale and coworkers [8] reported the novel synthesis of pyrones from alkynyl oxetanes in the presence of Au catalyst (Scheme 1). The striking feature in this report is the remarkable effect of the $\mathrm{Au}$ catalyst as well as the solvent on the reaction. When dichloromethane is used as solvent the formation of major product is pyrone with the carboxylic acid as byproduct. In the presence of acetonitrile there was no trace of the major product instead the decarboxylation occurs, while toluene gives both pyrone and decarboxylation byproduct. The reason for such selectivity was attributed to the higher temperature conditions that facilitate decarboxylation. According to the proposed mechanism (Scheme 1), Au interacts with $\mathbf{1}$ and forms either an oxophilic (B) or a carbophilic (A) intermediate, in either case yielding the final product, pyrone (1-a). This process was shown to proceed either through the Hashmi type cyclization [6,8], thereby yielding $\mathbf{D}$, or through ring opening $(\mathbf{C})$ followed by ring closure (D). Apart from the formation of pyrone $\mathbf{B}$ yields carboxylic acid (1-c) and decarboxylated (1-d) byproducts.

In continuation to our interests in understanding the mechanism of organic reactions $[20,25]$ the present study is aimed to unravel the various factors that underlie the formation of product and the byproduct during the course of reaction. This study aims to understand the preferred mechanism amongst the various possible reaction paths, the role of solvent which was shown to drive the product formation and also the effect of catalyst on the reaction path. Various other possible routes for the formation of the final products as well as byproducts were looked at.

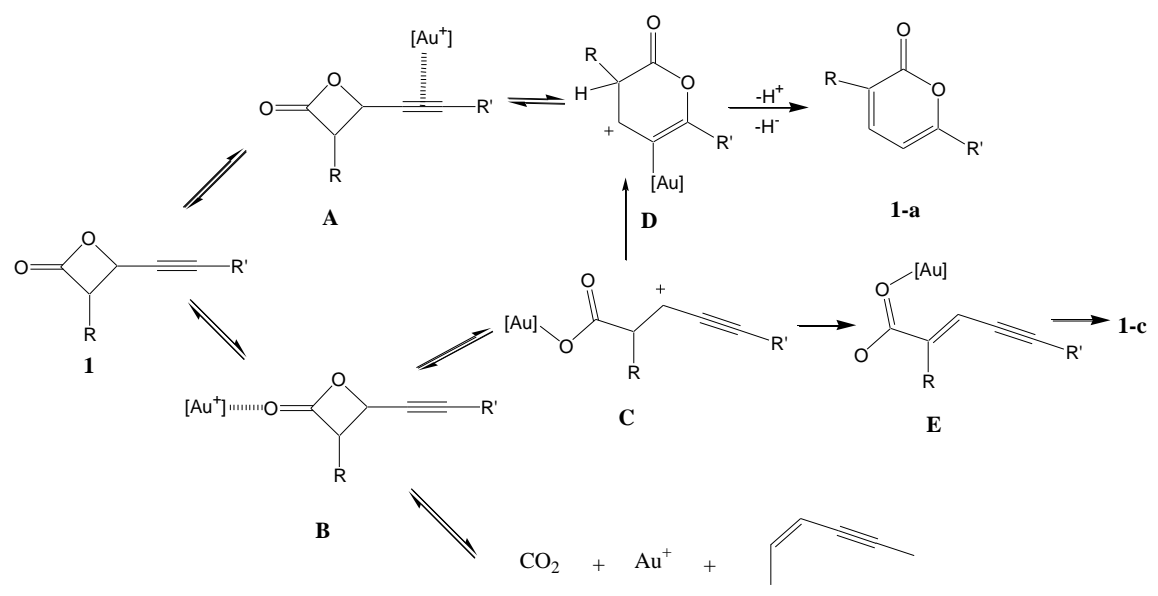

Scheme 1. Schematic representation of the proposed pathways. 


\section{Methodology}

Geometry optimizations were carried out on the reactants, intermediates, transition states and products using hybrid density functional theory, B3LYP. Double $\zeta$ basis set, LanL2DZ, was used for the metal, Au, as it considers relativistic effective core potential (ECP) [26-28], and 6-31G(d) basis set was used for all other atoms. Frequency calculations affirm the reactants, intermediates and products with zero imaginary frequency and transition states with one imaginary frequency with the corresponding normal mode. Solvent effects [29] were analyzed by optimizing the structures using polarizable continuum model (PCM). The solvents considered were dichloromethane, acetonitrile and toluene similar to those considered by Pale and coworkers. Atoms in molecules analysis is carried out using AIM2000 [30]. All the calculations are performed using G09 programme package [31].

\section{Results and Discussion}

The results are presented in two sections. In the first section the preferred mechanism among the various possible routes for the formation of products and byproducts considering the gas phase reactions is discussed. The second section deals with the solvent phase reaction profiles, generated for the preferred routes, and addresses the probable reasons for the product selectivity.

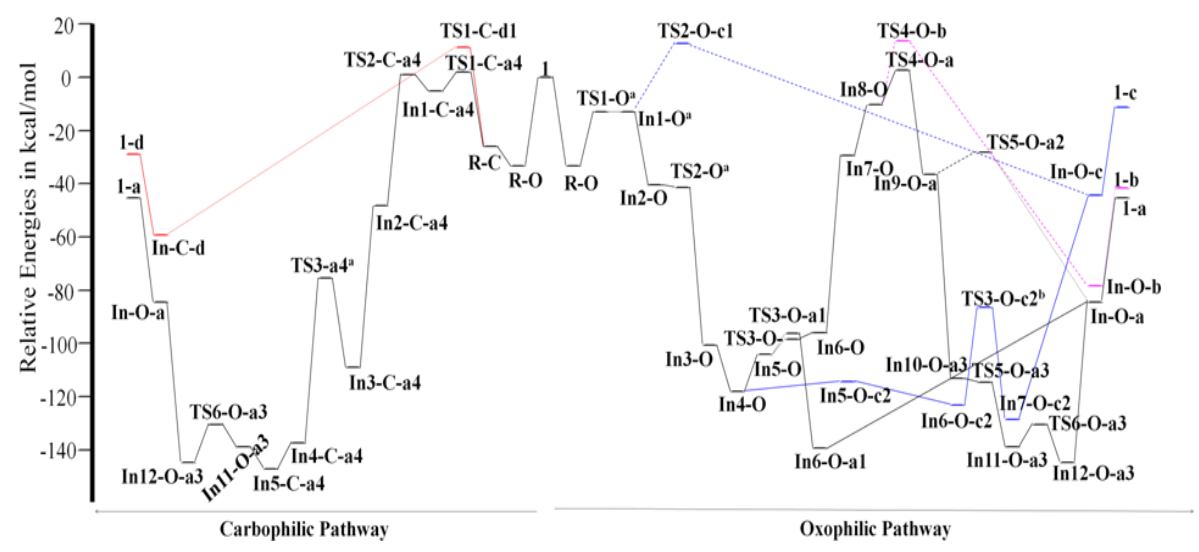

Fig. 1. $\mathrm{AuPH}_{3}$ catalyst mediated reaction energy profile generated using gas phase at B3LYP/6$31 \mathrm{G}(\mathrm{d})$ :LanL2DZ for Au level of theory (values with superscript ' $\mathrm{a}$ ' are obtained at B3LYP/6$31 \mathrm{G}(\mathrm{d})$ :LanL2DZ for Au//B3LYP/LanL2DZ level of theory and with ' $\mathrm{b}$ ' are obtained by performing constraint optimization).

The gas phase reaction profiles and the optimized structures are depicted in Figs. 1 and 2 , respectively. The attack of catalyst on the reactant $\mathbf{R}$ generates oxophilic (R-O) and carbophilic (R-C) reaction complexes. However, the energy difference between R-O and 
R-C is $7.3 \mathrm{kcal} / \mathrm{mol}$, former being lower in energy. Thus the reaction complex R-O should initially be formed and then undergo a crossover to form R-C. Then we proceeded to look at the formation of pyrone considering 1) the ring opening followed by ring closure and 2) 1,3-oxygen shift or Hashmi type cyclization mechanisms. R-O undergoes O-C bond cleavage forming In1-O via TS1-O. The attack of counterion on In1-O yields intermediate In2-O which then results in a proton removal transition state, TS2-O, in turn forming intermediate In4-O via In3-O.

In the absence of counterion In1-O can undergo a direct proton migration from ' $\mathrm{CH}_{2}$ ' to 'O', via TS2-O-c1 resulting in the formation of carboxylic acid product 1-c via In-O-c. The activation free energy barrier for TS2-O-c1 is $25.7 \mathrm{kcal} / \mathrm{mol}$ while the attack of counterion on In1-O lowers the energy by $27.5 \mathrm{kcal} / \mathrm{mol}$. Thus even before the reaction takes a different route counterion attacks the intermediate In1-O in turn forming In2-O. The protonated counterion, OTfH, then attacks the intermediate In4-O forming intermediate In5-O. The attack of counterion can occur either on the terminal carbon or on the carbon adjacent to it. The latter case results in a TS3-O-a1 which involves both proton migration and C-O bond formation in a single step thereby resulting in In5-O-a1 which after the removal of catalyst forms the final product. The attack of OTfH on the terminal carbon of In5-O proceeds via TS3-O and forms intermediate In6-O which further forms In7-O. In7-O rearranges itself in turn forming In8-O. This intermediate has a propensity to undergo either six-membered ring closure forming intermediate In9-O-a via TS4-O-a or a five-membered ring closure forming In-O-b via TS4-O-b. TS4-O-b is comparatively higher in energy than TS4-O-a by $13.9 \mathrm{kcal} / \mathrm{mol}$ suggesting the feasibility of 6-membered ring formation. Thus though theoretically five membered ring formation is possible the higher activation and reaction energies rule out its formation. In9-O-a can inturn form 1-a via In-O-a either in a single step which involves the direct proton migration TS, TS5-O-a2, or in multi steps involving counterion. The counterion abstracts the proton in In10-O-a3 via TS4-O-a3 and results in In11-O-a3 which is then reattacked by the protonated counterion forming In12-O-a2 via TS5-O-a3 and finally yields 1-a. Since the path preferred for the formation of pyrone depends on the TS3-O and TS3-O-a1 the activation free energy barriers for these two TSs are compared with each other. The barriers for TS3-O-a1 and TS3-O are -96.3 and $-98.3 \mathrm{kcal} / \mathrm{mol}$ respectively. The 2 $\mathrm{kcal} / \mathrm{mol}$ difference between these two TSs suggests the feasibility of both the TSs. However the later TSs and intermediates from In6-O are much higher in energy, 60 to $90 \mathrm{kcal} / \mathrm{mol}$. Hence the formation of final product, pyrone, must result via TS3-a1. The intermediate In4-O can undergo a bond rotation and form In5-O-c2 which opens up a path for the formation of carboxylic acid. The counterion OTfH attacks the ' $O$ ' proceeding to the carboxylic acid adduct, 1-c via TS3-O-c2 and In7-O-c2. The formation of pyrone can also proceed through 1,3-oxygen shift (Scheme 1). Previous reports on the ring expansion of cyclopropanols suggest a single step for the ring formation [17]. Initially the corresponding transition state which involves the simultaneous breakage of $\mathrm{O}-\mathrm{C}_{1}$ bond and the formation of $\mathrm{O}-\mathrm{C}_{2}$ bond was looked at, which led to futile attempts. So a different route was examined which involves the stepwise cyclization process wherein 
the R-C initially forms a bicylic intermediate In1-C-a4 via TS1-C-a4 which involves the attack of distal carbon on the oxygen thereby forming an $\mathrm{O}-\mathrm{C}_{2}$ bond.
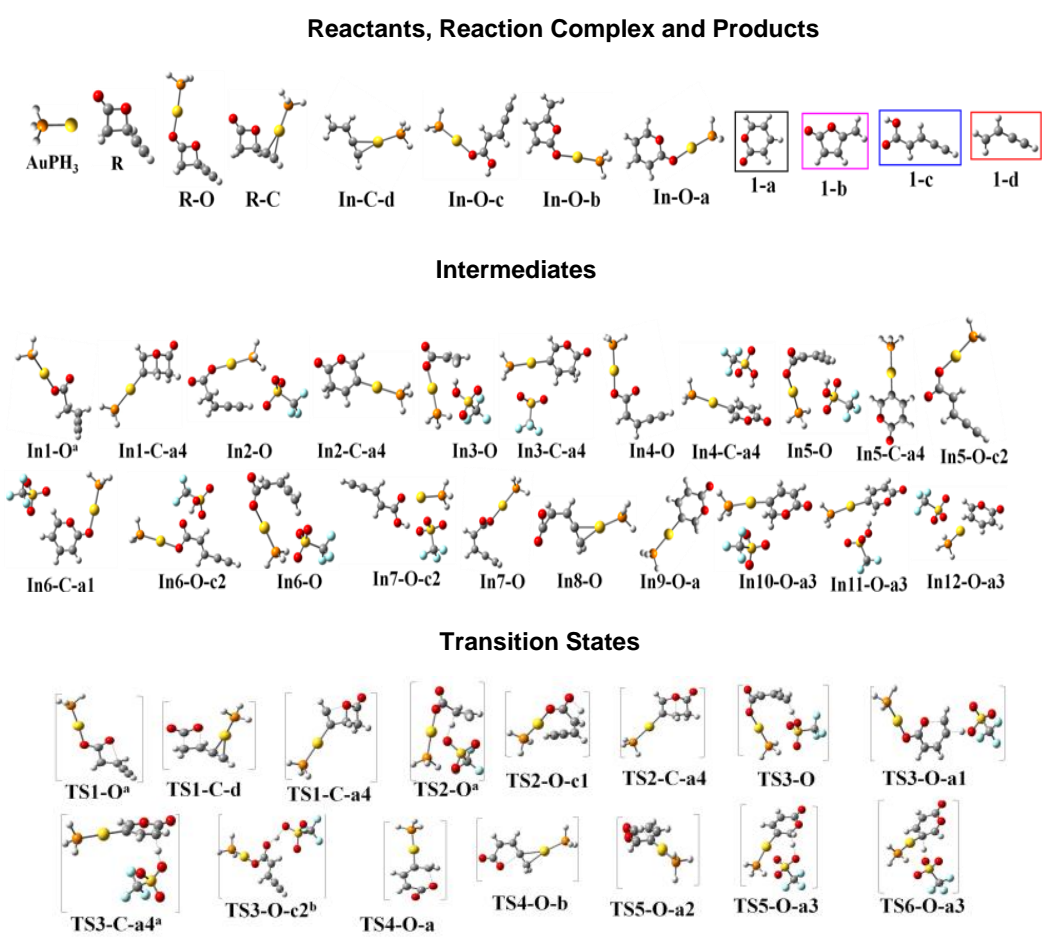

Fig. 2. Optimized structures of the reactants, reaction complexes, intermediates, transition states and products generated using gas phase at B3LYP/6-31G(d):LanL2DZ for Au level of theory (values with superscript ' $a$ ' are obtained at B3LYP/6-31G(d):LanL2DZ for Au//B3LYP/LanL2DZ level of theory and with ' $b$ ' are obtained by performing constraint optimization).

In1-C-a4 then undergoes $\mathrm{O}_{1} \mathrm{C}_{1}$ bond cleavage forming In2-C-a4, via TS2-C-a4, and then forms In3-C-a4 by the attack of counterion and in turn results in a proton elimination intermediate In4-C-a4 via TS3-a4 and later forms In5-C-a4. In5-C-a4 is reattacked by OTfH forming In11-O-a3 which finally yields 1-a via TS6-a3. From the above analysis it is observed that the key steps for the formation of the products are the crossover from $\mathbf{R}$ $\mathbf{O}$ to R-C. The preferred routes are depicted with thick lines in Fig 1 which are considered for further discussion to understand the solvent effects. Then the decarboxylation reaction was looked at. As reported by the authors [8] we initially tried to look at the transition states and intermediates for the decarboxylation path by considering R-O which led to futile attempts. However a [2+2] retroaddition TS, TS1-d1, could be obtained from reaction complex R-C which proceeds through a decarboxylation path and forms 1-d via In-C-d. Thus the formation of 1-d might proceed through the $[2+2]$ retroaddition transition state. 
After looking at the various possible routes for the formation of products we aim to study the effect of solvents on the preferred reaction paths as a huge solvent dependency was observed [8]. The relative energies for the TSs, intermediates and final products obtained using the solvents acetonitrile, dichloromethane and toluene are illustrated in Table 1. A quick look at the relative energies suggests that toluene drastically lowers the energies, which is followed by dichloromethane and then acetonitrile. The energy difference between the structures obtained using dichloromethane and acetonitrile as solvents are of the range $2-10 \mathrm{kcal} / \mathrm{mol}$ while the differences for the same with toluene are of the range $2-50 \mathrm{kcal} / \mathrm{mol}$. Thus in accordance with the experimental reports toluene is found to be a better choice among the solvents considered. The crossover of R-O to R-C bears a free energy difference of 5.3, 2.4 and $1.2 \mathrm{kcal} / \mathrm{mol}$ for toluene, dichloromethane and acetonitrile. As observed in the gas phase reactions the formation of pyrone proceeds either from the oxophilic intermediate R-O or the carbophilic intermediate R-C. Irrespective of the solvent considered the formation of ring opening intermediate, In1-O, is a feasible process, while the next step i.e. the counterion attack thereby forming intermediate In3-O is a crucial step. In the presence of acetonitrile the activation barrier is more than $\sim 100 \mathrm{kcal} / \mathrm{mol}$, while in the presence of dichloromethane and toluene the barrier drastically falls to $\sim 45$ and $\sim 14 \mathrm{kcal} / \mathrm{mol}$, respectively. This suggests that the attack of counterion is the rate determining step for formation of pyrone. The $\sim 100$ $\mathrm{kcal} / \mathrm{mol}$ energy barrier for TS2-O and inturn the formation of 1-a from 2-O can be ruled out for acetonitrile. The difference in the barrier between the two TSs TS3-O and TS3-Oa1 is more than $2 \mathrm{kcal} / \mathrm{mol}$, both involving the attack of HOTf. This suggests that the formation of pyrone from 5 proceeds via a multistep process rather than a single step. The crossover intermediate In5-O-c2 yielding 1-c is endothermic by about $10 \mathrm{kcal} / \mathrm{mol}$ irrespective of the solvent used which correlates with the yield obtained for the carboxylic acid byproduct. The carbophilic intermediate R-C can also proceed via the Hashmi type cyclization process and inturn form 1-a or undergo decarboxylation forming 1-d. These two TS (TS1-C-a4 and TS1-C-d1) do not differ much in the free energy barrier and suggest the feasibility of both the processes. From the reaction energy profiles it is clear that the free energy barriers for the reaction paths considered is not alone sufficient in understanding the preferred formation of the one product over other and suggests a more detailed understanding of the reaction.

Table 1. Relative energies for transition states, intermediates and products obtained at B3LYP level using LanL2DZ for Au and 6-31G(d) for all other atoms using PCM solvent conditions.

\begin{tabular}{lccc}
\hline & Acetonitrile & Dichloromethane & Toluene \\
\hline 1 & 0.0 & 0.0 & 0.0 \\
R-O & -6.4 & -8.8 & -17.2 \\
R-C & -5.2 & -6.3 & -12.0 \\
\hline Pyrone Formation & & & \\
TS1-O & & 12.7 & 8.3 \\
In1-O & 15.1 & 12.1 & 2.4 \\
In2-O $^{\mathrm{a}}$ & 14.7 & 56.7 & 23.0 \\
$\mathrm{TS}^{\mathrm{a}}-\mathrm{O}^{\mathrm{a}}$ & 11.2 & 61.9 & 25.2 \\
\hline
\end{tabular}




\begin{tabular}{lccc}
\hline In3-O & -43.4 & 1.7 & -34.0 \\
In4-O & -9.6 & -18.1 & -52.4 \\
In5-O & -46.4 & -1.0 & -37.8 \\
TS3-O & 12.8 & 3.8 & -31.4 \\
TS3-O-a1 & 16.4 & 6.9 & -28.9 \\
In6-O-a1 & -45.5 & -52.9 & -82.1 \\
In6-O & -42.2 & 1.8 & -31.4 \\
In7-O & -1.6 & -3.9 & -12.0 \\
In8-O & 8.3 & 6.9 & 1.5 \\
TS4-O-a & 23.0 & 21.3 & 16.6 \\
In9-O-a & -15.7 & -16.5 & -23.1 \\
In10-O-a3 & -61.3 & -15.0 & -48.3 \\
TS5-O-a3 & -6.6 & -15.0 & -49.1 \\
In11-O-a3 & -77.1 & -32.3 & -70.2 \\
TS6-O-a3 & -19.4 & -28.6 & -65.2 \\
In12-O-a3 & -91.1 & -44.9 & -78.8 \\
In-O-a & -56.5 & -59.5 & -69.3 \\
1-a & -44.9 & -45.0 & -45.1 \\
TS1-C-a4 & 27.6 & 25.4 & 16.9 \\
In1-C-a4 & -23.4 & 22.4 & 12.2 \\
TS2-C-a4 & 27.6 & 25.4 & 17.2 \\
In2-C-a4 & -23.4 & -25.1 & -33.1 \\
In3-C-a4 & -47.1 & -21.3 & -54.2 \\
TS3-C-a4 & 22.0 & 25.0 & -7.9 \\
In4-C-a4 & -75.4 & 31.0 & -68.4 \\
In5-C-a4 & -35.1 & -44.2 & -80.6 \\
\hline Decarboxylation product & & & \\
TS1-C-d & 28.9 & 28.1 & -4.1 \\
In-C-d & -37.0 & -39.6 & -45.9 \\
1-d & -26.4 & -26.7 & -27.6 \\
\hline Carboxylic acid formation & & & -23.1 \\
In5-O-c2 & -7.8 & -16.0 & -60.8 \\
In6-O-c2 & -69.2 & 10.7 & -27.4 \\
TS3-O-c2 & 19.0 & -24.5 & -10.9 \\
In7-O-c2 & -69.5 & -18.8 & \\
In-O-c & -16.6 & -10.4 & \\
1-c & -10.3 & & \\
\hline & & & \\
& & & \\
\hline & & & \\
\hline
\end{tabular}

In order to understand the reaction trends, two aspects were considered for this analysis 1) the crossover between the oxophilic and carbophilic intermediates and 2) the effect of solvent on these intermediates. The lower free energy difference of $1.2 \mathrm{kcal} / \mathrm{mol}$ in the presence of acetonitrile results in a rapid interconversion or crossover between the two isomers while the same requires a free energy of 5.3 and $2.4 \mathrm{kcal} / \mathrm{mol}$ for toluene and dichloromethane. From this it is clear that the formation of $\mathbf{R}-\mathbf{C}$ is easy in the presence of acetonitrile while the same is difficult in the presence of dichloromethane and toluene. It should be noted that in the presence of toluene reflux conditions during the reaction might decrease the crossover free energy and result in the rapid interconversion between the two reaction complexes which might open a way for the formation of 1-d. Understanding the huge effect of solvent on the reaction we performed solvent calculations using explicit solvent molecule in each case on the R-C as this can clearly suggest the formation 
decarboxylation byproduct. Various conformations were considered in each case and the lowest free energy structure is considered for further study. To look for any existing noncovalent interactions between the solvent and the intermediate atoms in molecules analysis was performed. The main idea behind this study is that if the solvent molecule involves in any non-covalent interactions with the $\mathrm{O}$ attached to the ring carbon then it is easier for the decarboxylation step to take place. The optimized structures showing the bond critical points along with the relative energies for most stable conformations of R-C are depicted in Fig 3. In the presence of acetonitrile R-C-A-1 with the solvent molecule attached to the $\mathrm{O}$ attached to the ring carbon is more stable than R-C-A-2 by $6.6 \mathrm{kcal} / \mathrm{mol}$. Also ring critical points suggest more interactions in R-C-A-1 than its counterpart. The decarboxylation product from R-C-D-1 intermediate is much easier. While the reverse is true for the dichloromethane solvent in which case the isomer R-C-D-1 is less stable than R-C-D-2 by $7.3 \mathrm{kcal} / \mathrm{mol}$. The ring critical points in this case suggest more interactions in R-C-D-2 than R-C-D-1. Thus in the presence of dichloromethane the decarboxylation step must be less feasible. Similar results are observed considering explicit toluene as solvent. The most stable isomer among the 4 isomers considered in the presence of toluene is R-C-T-1 which involves the interactions between the solvent and terminal carbon of alkyne and also $\mathrm{H}$ of $\mathrm{CH}_{2}$ group. However it can be observed that the isomer $\mathbf{R}$ C-T-2 is only $2.2 \mathrm{kcal} / \mathrm{mol}$ higher in energy compared to R-C-T-1.

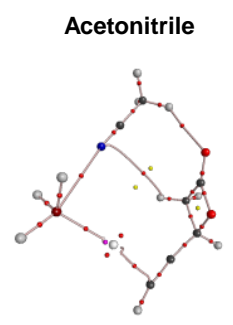

R-C-A

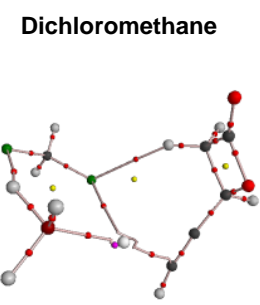

R-C-D

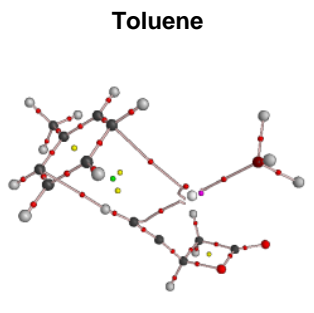

R-C-T

Fig. 3. Bond critical points for R-C involving explicit solvent molecules under PCM conditions using acetonitrile (A), dichloromethane (D) and toluene (T).

Comparing the reaction complex for 3 solvents, the reaction complex, in the presence of acetonitrile as solvent, possess more number of ring critical points suggesting weak interactions between the solvent and the ' $\mathrm{O}$ ' attached to the ring carbon thereby stabilizing the R-C complex. Hence once the R-C complex is formed the propensity for formation of decarboxylation transition state increases as the transition state formed during the process must be stabilized due to the non-covalent interactions. This in turn suggests that in case of acetonitrile the non-covalent interactions between solvent opens the way for the formation of decarboxylation product. Thus the energy barrier for crossover between the oxophilic and carbophilic intermediates and non-covalent interactions with the solvent molecule play a key role in the formation of final product. 


\section{Conclusion}

The cycloisomerization reaction from lactones to pyrones is studied theoretically. The crossover reaction from oxophilic to alkynophilic intermediate is a crucial step and the rate at which the crossover takes place determines the reaction path and inturn the formation of product. The proton elimination step, TS2-O, is observed to be the rate determining step for the formation of the final product. The rate determining step in toluene is much lower than in dichloromethane which reflects the observed experimental reports where the reaction is much faster in toluene than in dichloromethane. Atoms in molecules analysis on R-C using the explicit solvent model locates the bond critical points connecting the catalyst, solvent and the $\mathrm{CO}_{2}$ moiety of the lactone. The crossover free energy and the AIM analysis suggest the exclusive formation of decarboxylation product in acetonitrile solvent. The reaction path for the $\mathrm{AuCl}_{3}$ catalyzed reaction is found to be an uphill process which attributes to the low yields observed experimentally. The theoretical studies gave a new insight in understanding the reactivity. The results corroborate well with the observed experimental trends and suggest that the crossover between oxophilic to alkynophilic intermediates as well as non-covalent interactions between the reaction complex and solvent play a crucial role in the driving the reaction path.

\section{Acknowledgment}

Author thanks G. N. Sastry, Director, CSIR-NEIST for his guidance in manuscript preparation and for the computational facilities.

\section{References}

1. A. Corma, A. Leyva-Perez, and M. Sabater, J. Chem. Rev. 111, 1657 (2011). https://doi.org/10.1021/cr100414u

2. A. Nijamudheen and A. Datta, Chem. Eur. J. 26, 1442 (2020). https://doi.org/10.1002/chem.201903377

3. N. Krause and C. Winter, Chem. Rev. 111, 1994 (2011). https://doi.org/10.1021/cr1004088

4. A. S. K. Hashmi, Angew. Chem. Int. Ed. 49, 5232 (2010). https://doi.org/10.1002/anie.200907078

5. D. Pflästerer and A. S. K. Hashmi, Chem. Soc. Rev. 45, 1331 (2016). https://doi.org/10.1039/C5CS00721F

6. A. S. K. Hashmi and P. Sinha, Adv. Synth. Catal. 346, 432 (2004). https://doi.org/10.1002/adsc.200303201

7. A. Blanc, K. Tenbrink, J-M. Weibel, and P. Pale, J. Org. Chem. 74, 5342 (2009). https://doi.org/10.1021/jo9008172

8. T. Dombray, A. Blanc, J. Weibel, and P. Pale, Org. Lett. 12, 5362 (2010). https://doi.org/10.1021/jo9008172

9. A. G. Perez, C. S. Lopez, J. Marco-Contellas, O. N. Faza, E. Soriano, and A. R. de Lera, J. Org. Chem. 74, 2982 (2009). https://doi.org/10.1021/jo802516k

10. A. R. Jagdale and S. W. Youn, Eur. J. Org. Chem. 2011, 3904 (2011). https://doi.org/10.1002/ejoc.201100113 
11. M. Jia and M. Bandini, ACS Catal. 5, 1638 (2015). https://doi.org/10.1021/cs501902v

12. R. Zhu, D. Zhang, J. Guo, J. Mu, C. Duan, and C. Liu, J. Phys. Chem. A 114, 4689 (2010). https://doi.org/10.1021/jp100291c

13. K. Theilacker, H. B. Schlegel, M. Kaupp, and P. Schwerdtfeger, Inorg. Chem. 54, 9869 (2015). https://doi.org/10.1021/acs.inorgchem.5b01632

14. C. M. Krauter, A. S. K. Hashmi, and M. Pernpointner, Chem. Catal. Chem. 2, 1226 (2010). https://doi.org/10.1002/cctc.201000136

15. J. J. Varghese and S. H. Mushrif, React. Chem. Eng. 4, 165 (2019). https://doi.org/10.1039/C8RE00226F

16. Y. Yang, Y. Liu, R. Zhu, C. Liu, and D. Zhang, J. Org. Chem. 84, 9705 (2019). https://doi.org/10.1021/acs.joc.9b01441

17. T. L. Sordo and D. Ardura, Eur. J. Org. Chem. 2008, 3004 (2008). https://doi.org/10.1002/ejoc.200800043

18. Y. Yamamoto, J. Org. Chem. 72, 7817 (2007). https://doi.org/10.1021/jo070579k

19. A. Nijamudheen, D. Jose, and A. Datta, J. Phys. Chem. C 115, 2187 (2011). https://doi.org/10.1021/jp1101384

20. N. T. Patil, V. S. Raut, V. S. Shinde, G. Gayatri, and G. N. Sastry, Chem. Eur. J. 18, 5530 (2012). https://doi.org/10.1002/chem.201103668

21. A. S. Mahadevi and G. N. Sastry, Chem. Rev. 116, 2775 (2016). https://doi.org/10.1021/cr300222d

22. T. Ghebreghiorgis, B. Biannic, B. H. Kirk, D. H. Ess, and A. Aponick, J. Am. Chem. Soc. 134, 16307 (2012). https://doi.org/10.1021/ja306333a

23. A. J. Neel, M. J. Hilton, M. S. Sigman, and F. D. Toste, Nature 543, 637 (2017). https://doi.org/10.1038/nature21701

24. T. Pal, M. Alam, J. Hossen, S. Paul, H. Ahmad, and M. Sheikh, J. Sci. Res. 10, 291 (2018). https://doi.org/10.3329/jsr.v10i3.36379

25. G. Gayatri and G. N. Sastry, J. Phys. Chem. A 113, 12013 (2009). https://doi.org/10.1021/jp9038072

26. P. Pyykko, Chem. Soc. Rev. 37, 1967 (2008). https://doi.org/10.1039/B708613J

27. P. Pyykko, Angew. Chem. Int. Ed. 43, 4412 (2004). https://doi.org/10.1002/anie.200300624

28. D. J. Gorin and F. D. Toste, Nature 446, 395 (2007). https://doi.org/10.1038/nature05592

29. J. Tomasi, B. Mennucci, and R. Cammi, Chem. Rev. 105, 2999 (2005). https://doi.org/10.1021/cr9904009

30. F. Biegler-König and J. Schönbohm, AIM 2000 Version 2.0 (University of Applied Science, Germany, 2002).

31. M. J. Frisch, G.W. Trucks, H. B. Schlegel, G. E. Scuseria, M. A. Robb, J. R. Cheeseman, G. Scalmani, V. Barone, B. Mennucci, G. A. Petersson, H. Nakatsuji, M. Caricato, X. Li, H. P. Hratchian, A. F. Izmaylov, J. Bloino, G. Zheng, J. L. Sonnenberg, M. Hada, M. Ehara, K. Toyota, R. Fukuda, J. Hasegawa, M. Ishida, T. Nakajima, Y. Honda, O. Kitao, H. Nakai, T. Vreven, Jr. J. A. Montgomery, J. E. Peralta, F. Ogliaro, M. Bearpark, J. J. Heyd, E. Brothers, K. N. Kudin, V. N. Staroverov, R. Kobayashi, J. Normand, K. Raghavachari, A. Rendell, J. C. Burant, S. S. Iyengar, J. Tomasi, M. Cossi, N. Rega, J. M. Millam, M. Klene, J. E. Knox, J. B. Cross, V. Bakken, C. Adamo, J. Jaramillo, R. Gomperts, R. E. Stratmann, O. Yazyev, A. J. Austin, R. Cammi, C. Pomelli, J. W. Ochterski, R. L. Martin, K. Morokuma, V. G. Zakrzewski, G. A. Voth, P. Salvador, J. J. Dannenberg, S. Dapprich, A. D. Daniels, O. Farkas, J. B.

Foresman, J. V. Ortiz, J. Cioslowski, and D. J. Fox, Gaussian, Inc., Wallingford CT, 2009 Gaussian 09, Revision A.02. 